\title{
Geschichte der Medizin.
}

\section{Der Zwickauer Stadtarzt Dr. Sixtus Kolbenschlag.}

\author{
Von Lic. Dr. Otto Clemen in Zwickau.
}

Schon ofters ist auf die Tatsache hingewiesen worden, daß 3 in der ersten Hälfte des 16. Jahrhunderts eine ganz auffällig große Zahl wissenschaftlich bedeutender Männer aus den Mauern Zwickaus hervorgegangen ist. Auch unter den Stadtärzten jener Zeit ragten mehrere durch ungewöhnliche Tüchtigkeit hervor. Ueber den größten unter ihnen, Janus Cornarius, dessen „Bemühungen um die Wiederherstellung der durch den Arahismus verunstalteten griechischen Medizin" jetzt noch gerïhmt werden, ${ }^{1}$ ) bereite ich eine Monographie vor; über S te phan Wild, der 1526 als ",des Radts artz" angestellt wurde, besonders 1529 , als auch Zwickau von der Schweißsucht heimgesucht wurde, sich bewährte, 1534 aber das Physikat niederlegte, da er zum Leibarzt des Kurfürsten Johann Triedrich von Sachsen berufen worden war, hat kïrzlich Nikolaus Müller ${ }^{2}$ ) gehandelt. Hier möchte ich auf Sixtus Kolbenschlag, der in den letzten Jahren seiner Zwickauer Amtstätigkeit noch Wilds Kollege gewesen ist, die Aufmerksamkeit lenken, zumal da eine Veröffentlichung von ihm, ein 1525 in Zwickau gedrucktes Pestregiment, der Vergessenheit entrissen zu werden verdient.

Nur wenig wissen wir von seinen Lebensschicksalen. Er taucht zuerst auf in den ,Acta nationis Germanicae universitatis Bononiensis"; im Jahre 1517 wurde er als Ehrenmitglied in die Korporation aufge-

1) A. Hirsch, Allgemeine deutsche Biographie, Bd. 4, S. 481. 2) Archiv für Reformationsgeschichte, Bd. 7, S. $393 \mathrm{ff}$. 


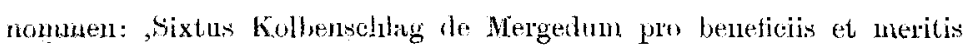
in nationen nostranl collatis incorporatus fuit'.1) Aus diesem Eintrag lernen wir ancl seine Heinat kemnen: er stannte ans Mergenthein in Fraukell. All 2. Januar 1518 wurde er dann zunı Doctor artiun et 1nedicinac promoviert. ${ }^{2}$ ) Nicht lange darauf wird er nacl Dentsclland zurückgekehrt sein. Im August 1522 wurde er init 40 Gulden Gehalt, freier Wohnung und Heizung vom Zwickauer Rate als Physikus angestellt. ${ }^{3}$ ) All 15. Juni 1524 heiratete er die Tochter des Anfang Mai 1520 verstorbenen Bürgermeisters Michel Rang, anı 6. August 1527 heiratete er, naclidem ihnı am 13. Januar 1526 seine erste Frau gestorben war, zull zweiten Male, und zwar "Gregor losen Tochter". ${ }^{4}$ )

Für seinespïteren Lebensschicksale habe ich im Zwickauer Ratsarchiv nur eine sie belenchtende Urkunde finden können. Fs ist ein in Fulda Montaks nacl Jacobi Apostoli (=28. Juli) 1533 aufgenommenes Protokoll, in dell Abt Johann von Fulda bekennt, daß heute vor ihm und seinen Hofräten sein Leibarzt Sixtus Kolbenschlag mit seinem ehlichen Gemah erschienen ist und einmütig bekannt haben, ,das sie mit Irer beyder wissen vund willen, Auch mit vorgehaptem rath ire behaußung zu Zwickaw in der Flaiscliergassen gelegenn, etwann Doctoris Stulers gewesenn, mit aller freihait vund gerechtigkait, wie sie es in vorzeitten ingeliapt, . . . Melchiorn von Creutzing, der rechten doctori" für $750 \mathrm{Gul}$ den verkauft haben. Danacl hat also Kolbenschlag das einst dem Zwickauer Stadtarzt und „Lïgenchronisten“ Erasmus Stella ${ }^{5}$ ) gehörige lireihaus in der Fleischergasse besessen, 1533 aber an den späteren Auntmann von Colditz Melchior von Creitzen ${ }^{6}$ ) verkauft. Kolbenschlag war damals Leibarzt des (evangelisch gesinnten) Fuldaer Abtes Johannes III. von Henneberg. ${ }^{7}$

Nur einmal, soviel ich weiß, ist Kolbenschlag als medizinischer Sclıriftsteller liervorgetreten. Sein dem Impressum zufolge am 20 . Fe bruar 1525 bei Jörg Gastel in Zwickau im Druck erschienenes Pestregiment ist bisher unbeachtet geblieben; weder in Panzers Annalen der älteren deutschen Literatur, noch in Wellers Repertorium typographicum, noch im Thesaurus libellorum historiam reformationis illu strantium von Weigel-Kuczynski noch sonstwo wird es erwähnt Fs ilmfaßt 18 Quartblätter, von denen das letzte leer ist. Der Tite lautet: „Eyn seer tröst-/ lich vnd kurtz Regiment wi / der die schweren vnd erschrecklichen / kranckheyt der Pestilentz, durch den / hoch gelartenn herrn Sixtum / Colbenschlag der freyen künst / vnd ärtzney Doctor begrif / fen, vnd dem Erbarn / Rath zu Zwickaw zu / sonderm I'rost be-/schriben. " Darunter sieht man das Zwickauer Stadt wappen mit den drei Schwänen, und umrahmt wird der Titel von einer lustigen Bordüre, die $\mathrm{G}$ astel oft gebraucht hat. ${ }^{8}$ ) Die Zwickauer Rats schulbibliothek besitzt zwei Exemplare des seltenen Drucks.

Kolbenschlag zeigt zuerst, „was Pestilenz sei und bedente“. Er wiederholt da einfach die Definitionen des Haliabbas, Placentinus, Isidorus. Ausführlicher behandelt er sodann die Entstehungsursachen der Krankheit. Sie komme aus entweder von oben herab oder auf der Frde oder aus der Erde oder aus dem Wasser heraus wehender verdorbener Luft. Indes seien inımer nur gewisse Menschen für die Pest prädisponiert. Er erklärt das dırch folgendes Gleichnis: „Im Herbst, so die ersten reyffen oder kelt kommen, hastu eynen schönen bawmgarten, am morgen felt eyn reyff zugleych auff die bewm alle, der eyn bawm würdt schwelck am lawb, der ander gantz dürre, der dritte behelt seyn grün. nun, das ist alles von eynem gleichen reyff, aber der reyff ist daran nit vrsach, sonder der bawm, wann (= denn) eyn bawm ist eyner andern art vnd natur wann der ander. Also in dysem lauff ist eyn mensch der ding entpfencklich vnd der ander nit." Auf viererle Weise könne die Luft verdorben werden: 1. ,so die vier zeyt jr art nit behaben", d. h. wenn ein der betreffenden Jahreszeit unnatürliches Wetter herrsche; wenn Sommer, Winter, Lenz und Herbst ,,jre natur behaben, so ist alles gesund, das do wechst vnd lebt, wann sie sich aber verkeren, so mag auch alleyn durch jre Wirckung sich das kindt inn mutter leyb verkeren biß auf den todt, an (= ohne) alle eusserlich zutuhn." 2. ändere sich die Luft ,durch die würckung alleyn der gestirn von oben herab, vnsichtbar vnsern augen, dauon die Astrologi vil schreyben." 3. „durch außriechung dem erdrich von feule odder böser ertz, aber

1) Gustav C. Knod, Deutsche Studenten in Bologna (1289 bis 1562). Biographischer Index $\mathrm{zu}$ den Acta nationis Germanicae universitatis Bononiensis. Berlin 1899, S. 701. - 2) Ebenda. - ${ }^{3}$ ) E'mil Herzog, Chronik der Kreisstadt Zwickall, Bd. 2, Zwickau 1845 S. 198. - t) Diese Angaben entnehme ich den Zwickauer Annalen l'eter Schumanns (Handschr. der Zwickauer Ratschulbibliothek). Dem Bürgermeister Rang war am 28. Februar 1520 ein Boin abgesclinitten worden; er starb am 4., 5. oder 6. Mai. - 5) Vgl. über ihn zuletzt meine Bemerkungen in den Mitteilungen des Altertumsveroin für Zwickau und Umgegend, Bd. 8, S. $177 \mathrm{ff}$. - Stella war am 2. April 1521, sein Weib am 19. März 1528 gestorben (Peter Schumann). $\left.{ }^{6}\right)$ Vgl. über ihn zuletzt Enders, Luthers Briefwechsel, Bd. 12, S. $244^{2}$.

ग) Enders, Bd. 7, S. $102^{1}$. - ${ }^{8}$ ) A. v. Dommer, Lutherdrucke auf der Hamburger Stadtbibliothek, 1516-1523, Leipzig 1888, S. 235, No. 70 C. A. Götze, Die liochdeutschen Drucker der Reformationszeit, Straßburg 1905, S. 94 , No. 109
( - oder) böser val vbel begrabuug der cörper, das dann eyn vagenessener schaden ist, vorauf iun zeyt der Pestilentz, anch zufluß vr. reyner ding oder heymlichel gemach, der gleychen mag auch lieinlich oder oftenbar vergifftung inm wassel beschehen, heymlich voln gestiru, offenber durch böß llischung oder evngiessing stinckender Ding, als flaclıs, hanff, dorinn rössen vnd feulen, do von die visch abstehell, für welchen man sich hiitten sol." 4 . verunreinigten die Menschell durch ihre Ausdünstung die Luft. Endlich aber miïsse man jede Pest anch als eine Strafe Gottes auffassen. Daher wolle er seine Mitbiirger er. mahnen, vor allen Dingen sich ,mit eynen simpeln gehorsanell vurl gantz vestem glauben" zu Gott zu kehren, von Sünden abzustehlien 1111 sich der rechten Nächstenliebe zu befleißigen zur Besänftigumg des Zornes Gottes. Freilich solle nuan darüber nicht die leiblich-natiirliche Hilfe vernachlässigen, denn Gott habe doch dic Wurzeln, Krä1ıter ,saulpt, andern Aromaten zll auffenthaltung vnserer natur" geschaffen. Mian solle sich auch nicht an die,,alten vnwitzigen weyber oder der ärtzney verschmelier vnd winckelärtzt" wenden, ,wann solche durch jr vermeßne vnwissende künheyt vil menschen inn dyser vnnd anderen vil kranckheyt aufs wenigst inn grösser wetagen bringen vnd zll zeyttell den grimmigen Todt selbst eyngeben."

Im dritten Kapitel verbreitet sich Kolbenschlag über die „Vorzeiclıen künftiger Pestilenz". Er erwähnt da zuerst feurige Wrsclieinungen am Himmel und in der Luft, besonders im Anfang des Herbstes. Ferner sei es bedenklich, wenn der Sommer nicht recht warm sei, anch wenig Regen und Wind bringe, dafür viel trïbe Luft und finstere, dicke, stinkende Nebel und Gewölk. ,Item, so vil vnzelicher vergiffter würm fnd thier erwachsen, alß Schlangen, Ratten, Meuß, Schuecken, Rauppen, Flygen vnd dergleychen." Auch tote Fische und Frösche im Wasser seien Unglücksboten.

Im vierten Kapitel gibt unser Autor die Symptome an, aus dellen zu erkennen sei, daß ein Mensch von der Pest befallen sei. Besonders: interessant ist es, daß er davor warnt, der Harnanalyse zuviel Wert beizulegen. ,ich hab das auclı selbst zum öftern mal in dreyen sterben vonn vilen nienschen gesehen, das die wasser außerwelt vnnd gutem harn gleych waren, vnd die menschen sturben doch inn kürtz, darumb gib nit alleyn glauben darauff, dn würst anders betrogen inn dyser vnd vil andern kranckheyten."

Im fünften Kapitel gibt Kolbenschlag Verhaltungsmaßregeln bein Ausbruch der Pest. Das beste sei die Wlucht, und zwar so bald als llöglich und je weiter, je besser. Wer aber zurückbleiben müsse, der solle sich vor Ansteckung hüten, darim, ,vermeyden gemeynscliafft des volcks, die gassen vnd stet, do vil volck handelt, alß offene märckt, Kaufflieuser, Bierheuser, Badstuben, Dantzheuser" u. dgl., aucl nichti essen und trinken mit Erkrankten, ,noch jre cleyder, betgewand nit gebrauchen," ferner etzliche bewährte Arzneien nehmen und eine bestimmte Diät einhalten.

Hierüber und zum Schlusse über die Behandlung der Bubonen spendet Kolbenschlag eingehende Belehrung, wobei er sich jedoch meist in ausgetretenen Geleisen bewegt und besonders von dem berühmten Leipziger. Medizinprofessor Heinlich Stromer voll Aner. bach ${ }^{1}$ ) abhängig erweist. 